PROCEEDINGS OF THE

AMERICAN MATHEMATICAL SOCIETY

Volume 128, Number 12, Pages 3553-3561

S 0002-9939(00)05449-6

Article electronically published on May 18, 2000

\title{
ON THE POINTWISE MAXIMUM OF CONVEX FUNCTIONS
}

\author{
S. P. FITZPATRICK AND S. SIMONS
}

(Communicated by Dale Alspach)

This paper is dedicated to Professor Robert Phelps

\begin{abstract}
We study the conjugate of the maximum, $f \vee g$, of $f$ and $g$ when $f$ and $g$ are proper convex lower semicontinuous functions on a Banach space $E$. We show that $(f \vee g)^{* *}=f^{* *} \vee g^{* *}$ on the bidual, $E^{* *}$, of $E$ provided that $f$ and $g$ satisfy the Attouch-Brézis constraint qualification, and we also derive formulae for $(f \vee g)^{*}$ and for the "preconjugate" of $f^{*} \vee g^{*}$.
\end{abstract}

\section{INTRODUCTION}

Let $E$ be a real nontrivial Banach space. If $f: E \rightarrow \mathbb{R} \cup\{\infty\}$, we write

$$
\operatorname{dom} f:=\{x \in E: f(x) \in \mathbb{R}\},
$$

the "effective domain" of $f$. We write $\mathcal{P C} \mathcal{L S C}(E)$ for the set of all convex lower semicontinuous functions $f: E \rightarrow \mathbb{R} \cup\{\infty\}$ such that $\operatorname{dom} f \neq \emptyset$. (The "P्P" stands for "proper", which is the adjective frequently used to denote the fact that the effective domain of a function is nonempty.)

We write $E^{*}$ for the dual space of $E$. If $f \in \mathcal{P C} \mathcal{L S C}(E)$, we define $f^{*}: E^{*} \rightarrow$ $\mathbb{R} \cup\{\infty\}$ by

$$
f^{*}\left(x^{*}\right):=\sup _{E}\left(x^{*}-f\right),
$$

the conjugate of $f$. Then (see [5], p. 210) $f^{*} \in \mathcal{P C} \mathcal{L S C}\left(E^{*}\right)$.

We define the biconjugate, $f^{* *}$, of $f$ by

$$
f^{* *}\left(x^{* *}\right):=\left(f^{*}\right)^{*}\left(x^{* *}\right) \quad\left(x^{* *} \in E^{* *}\right) .
$$

From what we have observed above, $f^{* *} \in \mathcal{P C} \mathcal{L S C}\left(E^{* *}\right)$. In fact, $f^{* *}$ is lower semicontinuous with respect to the weak ${ }^{*}$ topology of $E^{* *}$ and (see [5], p. 210 again)

$$
\text { for all } x \in E, \quad f^{* *}(\widehat{x})=f(x),
$$

where $\widehat{x}$ is the canonical image of $x$ in $E^{* *}$.

Received by the editors May 11, 1998 and, in revised form, January 29, 1999.

2000 Mathematics Subject Classification. Primary 46N10, 49J52, 49N15.

Key words and phrases. Banach space, convex function, conjugate, biconjugate, maximum, Attouch-Brézis constraint qualification, preconjugate. 
Let $f, g \in \mathcal{P C} \mathcal{L S C}(E)$. We say that $f$ and $g$ satisfy the Attouch-Brézis constraint qualification if

$$
\bigcup_{\lambda>0} \lambda(\operatorname{dom} f-\operatorname{dom} g) \text { is a closed subspace of } E .
$$

It is well known that if $f, g \in \mathcal{P C} \mathcal{L S C}(E)$ and $f$ and $g$ satisfy $(\mathcal{A B})$, then

$$
(f+g)^{* *}=f^{* *}+g^{* *} \text { on } E^{* *} .
$$

In fact, Rockafellar used the equality (0.2) (under a stronger constraint qualification) in his proof in [5], Proposition 1, pp. 211-212 that the subdifferential of an element of $\mathcal{P C} \mathcal{L S C}(E)$ is maximal monotone. The equality $(0.2)$ follows easily from the "inf-convolution" formula for $(f+g)^{*}$; namely that, for all $w^{*} \in E^{*}$,

$$
(f+g)^{*}\left(w^{*}\right)=\min _{y^{*}, z^{*} \in E^{*}, y^{*}+z^{*}=w^{*}}\left[f^{*}\left(y^{*}\right)+g^{*}\left(z^{*}\right)\right],
$$

which was established by Attouch-Brézis in [1], Corollary 2.3, pp. 131-132.

In this paper, we consider the corresponding problem with $f+g$ replaced by $f \vee g$, where, for all $f, g \in \mathcal{P C} \mathcal{L S C}(E), f \vee g$ is defined by

$$
(f \vee g)(x):=\max \{f(x), g(x)\} \quad(x \in E) .
$$

Indeed, we will prove in Theorem 6 that if $f, g \in \mathcal{P C} \mathcal{L S C}(E)$ and $f$ and $g$ satisfy $(\mathcal{A B})$, then

$$
(f \vee g)^{* *}=f^{* *} \vee g^{* *} \text { on } E^{* *}
$$

We will complement this in Remark 8 by giving an example showing that the equality (0.4) can fail when $(\mathcal{A B})$ is not satisfied, even if $f \vee g \in \mathcal{P C} \mathcal{L S C}(E)$. Now (0.4) would follow easily from the equality that, for all $w^{*} \in E^{*}$,

$$
(f \vee g)^{*}\left(w^{*}\right)=\inf _{\rho \in[0,1], u^{*}, v^{*} \in E^{*}, \rho u^{*}+(1-\rho) v^{*}=w^{*}}\left[\rho f^{*}\left(u^{*}\right)+(1-\rho) g^{*}\left(v^{*}\right)\right] .
$$

Unfortunately, (0.5) fails even if $E=\mathbb{R}^{2}, g \in \mathcal{C C}(E)$ and $f$ and $g$ satisfy $(\mathcal{A B})$, where $\mathcal{C C}(E)$ stands for the set of all real convex continuous functions on $E$. We give an example of this in Remark 3. The actual formula for $(f \vee g)^{*}$ is much more complicated. In fact, we give two such formulae. The first, in (2.3), appears in Traoré and Volle, [7, Section 7, p. 149 and does not seem to lead easily to (0.4). We now give the background for the second, much more complicated formula, which appears in (2.1), and does lead easily to (0.4). Let $F$ be a nontrivial Banach space. (The reason why we also introduce the symbol $F$ to represent a Banach space is that we will be applying these concepts with $F:=E^{*}$.) If $w \in F$ and $\delta>0$, let $B(w, \delta):=\{x \in F:\|x-w\|<\delta\}$ and $L(w, \delta):=\{(\rho, \sigma, u, v): \rho>0, \sigma>0, u, v \in F, \rho+\sigma=1, \rho u+\sigma v \in B(w, \delta)\}$.

Suppose that $f, g \in \mathcal{P C} \mathcal{L S C}(F)$. If $w \in F$, write

$$
\begin{gathered}
\left(f \hat{\delta}^{\prime} g\right)(w):=\inf _{(\rho, \sigma, u, v) \in L(w, \delta)}[\rho f(u)+\sigma g(v)] \quad(\delta>0) \quad \text { and } \\
(f \underset{0}{\wedge} g)(w):=\sup _{\delta>0}\left(f \wedge_{\delta} g\right)(w)=\lim _{\delta \rightarrow 0}\left(f \wedge_{\delta}^{\wedge} g\right)(w) .
\end{gathered}
$$

Then the formula that we shall give in (2.1) is that if $w^{*} \in E^{*}$, then

$$
(f \vee g)^{*}\left(w^{*}\right)=\left(f_{0}^{*} g^{*}\right)\left(w^{*}\right)
$$


Incidentally, the equality (0.4) is closely related to the result proved by Gossez in [3], Lemme 2.1, p. 375 that the subdifferential of an element of $\mathcal{P C} \mathcal{L C C}(E)$ is maximal monotone of "dense type". Unfortunately, it would take us much too far afield to dwell on this issue any further.

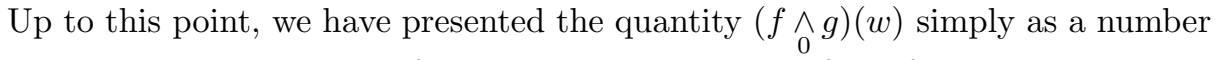
which appears as the result of certain computations. In fact, $f \underset{0}{\wedge} g$ has much more significance when we consider it as a function. We shall show in Theorem 11 that if $f, g \in \mathcal{P C} \mathcal{L S C}(F)$ and $\operatorname{dom} f^{*} \cap \operatorname{dom} g^{*} \neq \emptyset$, then

$$
f \underset{0}{\wedge} g \in \mathcal{P C L S C}(F) \text { and }(f \underset{0}{\wedge} g)^{*}=f^{*} \vee g^{*} \text { on } F^{*}
$$

In other words, $f \underset{0}{\wedge} g$ is the "preconjugate" of $f^{*} \vee g^{*}$. We shall use this result in Theorem 12 to give a precise description of when (0.4) occurs. Namely, if $f, g \in$ $\mathcal{P C} \mathcal{L S C}(E)$ and $\operatorname{dom} f \cap \operatorname{dom} g \neq \emptyset$, then (0.4) occurs if, and only if,

$$
(f \vee g)^{*}=f_{0}^{*} \underset{0}{\wedge} g^{*} \text { on } E^{*}
$$

In the proof of Theorem 2, we shall use the minimax theorem below, which follows from a result of Fan (see [2]). (See also [4] and [6] for simple generalizations of Fan's result.)

Theorem 1. Let $A$ be a nonempty convex subset of a vector space, and let $B$ be a nonempty compact convex subset of a topological vector space. Let $h: A \times B \rightarrow \mathbb{R}$ be convex on $A$, and concave and upper semicontinuous on $B$. Then

$$
\inf _{A} \max _{B} h=\max _{B} \inf _{A} h
$$

\section{The conjugate of a maximum}

Theorem 2. Suppose that $f, g \in \mathcal{P C} \mathcal{L} \mathcal{S C}(E), f$ and $g$ satisfy $(\mathcal{A B})$ and $w^{*} \in E^{*}$. Then:

$$
(f \vee g)^{*}\left(w^{*}\right)=\left(f_{0}^{*} \underset{\hat{0}}{\wedge} g^{*}\right)\left(w^{*}\right)
$$

Proof. We first prove that if $\rho, \sigma>0$, then there exist $u^{*}, v^{*} \in E^{*}$ such that

$$
\rho u^{*}+\sigma v^{*}=w^{*} \quad \text { and } \rho f^{*}\left(u^{*}\right)+\sigma g^{*}\left(v^{*}\right)=\sup _{A}\left[w^{*}-\rho f-\sigma g\right],
$$

where $A$ is the nonempty convex set $\operatorname{dom} f \cap \operatorname{dom} g$. To this end, let $\rho, \sigma>0$. Clearly $\rho f$ and $\sigma g$ also satisfy $(\mathcal{A B})$; consequently, from the Attouch-Brézis formula for the conjugate of a sum (see (0.3) above), there exist $y^{*} \in E^{*}$ and $z^{*} \in E^{*}$ such that

$$
y^{*}+z^{*}=w^{*} \quad \text { and } \quad(\rho f)^{*}\left(y^{*}\right)+(\sigma g)^{*}\left(z^{*}\right)=(\rho f+\sigma g)^{*}\left(w^{*}\right) .
$$

We now put $u^{*}:=y^{*} / \rho$ and $v^{*}:=z^{*} / \sigma$, and obtain (2.2) since we then have $(\rho f)^{*}\left(y^{*}\right)=\rho f^{*}\left(u^{*}\right),(\sigma g)^{*}\left(z^{*}\right)=\sigma g^{*}\left(v^{*}\right)$ and

$$
(\rho f+\sigma g)^{*}\left(w^{*}\right)=\sup _{A}\left[w^{*}-\rho f-\sigma g\right] .
$$

We next prove that

$$
(f \vee g)^{*}\left(w^{*}\right)=\min _{\lambda \in[0,1]} \sup _{A}\left[w^{*}-\lambda f-(1-\lambda) g\right] .
$$


This follows from the minimax theorem, Theorem 1 , with $B:=[0,1]$, since

$$
\begin{aligned}
(f \vee g)^{*}\left(w^{*}\right) & =\sup _{x \in A}\left[\left\langle x, w^{*}\right\rangle-(f \vee g)(x)\right] \\
& =\sup _{x \in A} \min _{\lambda \in[0,1]}\left[\left\langle x, w^{*}\right\rangle-\lambda f(x)-(1-\lambda) g(x)\right] .
\end{aligned}
$$

We now prove the inequality " $\geq$ " in (2.1). Since this is trivially true if $(f \vee g)^{*}\left(w^{*}\right)=\infty$, we can and will suppose that $(f \vee g)^{*}\left(w^{*}\right) \in \mathbb{R}$. Let $\delta, \varepsilon>0$. We shall prove that there exists $\left(\rho, \sigma, u^{*}, v^{*}\right) \in L\left(w^{*}, \delta\right)$ such that

$$
\rho f^{*}\left(u^{*}\right)+\sigma g^{*}\left(v^{*}\right) \leq(f \vee g)^{*}\left(w^{*}\right)+\varepsilon .
$$

The desired inequality will then follow by taking the infimum over $\left(\rho, \sigma, u^{*}, v^{*}\right) \in$ $L\left(w^{*}, \delta\right)$ and then letting $\delta \rightarrow 0$ and $\varepsilon \rightarrow 0$. From (2.3), there exists $\lambda \in[0,1]$ such that

$$
\sup _{A}\left[w^{*}-\lambda f-(1-\lambda) g\right]=(f \vee g)^{*}\left(w^{*}\right) .
$$

Case $1(\lambda \in(0,1))$. From $(2.2)$, there exist $u^{*}, v^{*} \in E^{*}$ such that

$$
\lambda u^{*}+(1-\lambda) v^{*}=w^{*} \text { and } \lambda f^{*}\left(u^{*}\right)+(1-\lambda) g^{*}\left(v^{*}\right)=(f \vee g)^{*}\left(w^{*}\right)
$$

and (2.4) is immediate with $\rho:=\lambda$ and $\sigma:=1-\lambda$.

Case $2(\lambda=0)$. Here we have

$$
\sup _{A}\left[w^{*}-g\right]=(f \vee g)^{*}\left(w^{*}\right) .
$$

As we have already observed, $f^{*} \in \mathcal{P C} \mathcal{L} \mathcal{S C}\left(E^{*}\right)$. Hence there exists $x^{*} \in E^{*}$ such that $f^{*}\left(x^{*}\right) \in \mathbb{R}$. If $\rho>0, \sigma>0, \rho+\sigma=1$ and $(\rho, \sigma)$ is sufficiently close to $(0,1)$, then

$$
\left(\rho, \sigma, x^{*}, w^{*}\right) \in L\left(w^{*}, \delta\right) \quad \text { and } \quad \rho f^{*}\left(x^{*}\right) \leq \rho(f \vee g)^{*}\left(w^{*}\right)+\varepsilon .
$$

Using (2.2) again, there exist $u^{*}, v^{*} \in E^{*}$ such that

$$
\rho u^{*}+\sigma v^{*}=\rho x^{*}+\sigma w^{*}
$$

and

$$
\begin{aligned}
\rho f^{*}\left(u^{*}\right)+\sigma g^{*}\left(v^{*}\right) & =\sup _{A}\left[\rho x^{*}+\sigma w^{*}-\rho f-\sigma g\right] \\
& =\sup _{A}\left[\rho\left(x^{*}-f\right)+\sigma\left(w^{*}-g\right)\right] \\
& \leq \rho \sup _{A}\left[x^{*}-f\right]+\sigma \sup _{A}\left[w^{*}-g\right] \\
& \leq \rho f^{*}\left(x^{*}\right)+\sigma \sup _{A}\left[w^{*}-g\right] .
\end{aligned}
$$

Thus, from (2.6) and (2.5),

$$
\begin{aligned}
\rho f^{*}\left(u^{*}\right)+\sigma g^{*}\left(v^{*}\right) & \leq\left[\rho(f \vee g)^{*}\left(w^{*}\right)+\varepsilon\right]+\sigma(f \vee g)^{*}\left(w^{*}\right) \\
& =(f \vee g)^{*}\left(w^{*}\right)+\varepsilon .
\end{aligned}
$$

We now obtain (2.4) since, from (2.6) and (2.7), $\left(\rho, \sigma, u^{*}, v^{*}\right) \in L\left(w^{*}, \delta\right)$.

Case $3(\lambda=1)$. The proof of this is similar to that of Case 2, except that the roles of $f$ and $g$ are reversed. This completes the proof of the inequality " $\geq$ " in (2.1). 
We now prove the reverse inequality. Let $x \in A$ and $\left(\rho, \sigma, u^{*}, v^{*}\right) \in L\left(w^{*}, \delta\right)$. Then

$$
\begin{aligned}
\rho f^{*}\left(u^{*}\right)+\sigma g^{*}\left(v^{*}\right) & \geq \rho\left[\left\langle x, u^{*}\right\rangle-f(x)\right]+\sigma\left[\left\langle x, v^{*}\right\rangle-g(x)\right] \\
& =\left\langle x, \rho u^{*}+\sigma v^{*}\right\rangle-\rho f(x)-\sigma g(x) \\
& \geq\left\langle x, w^{*}\right\rangle-\delta\|x\|-(f \vee g)(x) .
\end{aligned}
$$

Taking the infimum over $\left(\rho, \sigma, u^{*}, v^{*}\right) \in L\left(w^{*}, \delta\right)$, we obtain

$$
\left(f^{*} \underset{\delta}{\wedge} g^{*}\right)\left(w^{*}\right) \geq\left\langle x, w^{*}\right\rangle-\delta\|x\|-(f \vee g)(x) \text {. }
$$

Letting $\delta \rightarrow 0$

$$
\left(f^{*} \underset{0}{\wedge} g^{*}\right)\left(w^{*}\right) \geq\left\langle x, w^{*}\right\rangle-(f \vee g)(x) .
$$

The inequality " $\leq$ " in (2.1) now follows by taking the supremum of the right hand side over $x \in A$. (Note: this can also be deduced from Lemma 10(a), which is independent of the analysis in this Theorem.)

This completes the proof of Theorem 2.

If $C \subset E$, the indicator function of $C$ is the function $I_{C}: E \rightarrow \mathbb{R} \cup\{\infty\}$ defined by

$$
I_{C}(x):= \begin{cases}0 & \text { if } x \in C \\ \infty & \text { otherwise }\end{cases}
$$

Remark 3. We now give the promised example where $f, g \in \mathcal{P C} \mathcal{L S C}(E)$ and $f$ and $g$ satisfy $(\mathcal{A B})$, but $(0.5)$ fails. (We leave it to the reader to check that $(0.5)$ does hold if both $f \in \mathcal{C C}(E)$ and $g \in \mathcal{C C}(E)$.) Here is the example. Define $f \in \mathcal{P C} \mathcal{L} \mathcal{S C}\left(\mathbb{R}^{2}\right)$ and $g \in \mathcal{C C}\left(\mathbb{R}^{2}\right)$ by

$$
f\left(x_{1}, x_{2}\right):= \begin{cases}x_{2} & \text { if } x_{1} \geq 0 \\ \infty & \text { otherwise }\end{cases}
$$

and

$$
g\left(x_{1}, x_{2}\right):=x_{1} .
$$

Then $(f \vee g)^{*}(0)=-\inf (f \vee g)=0$. On the other hand, $f^{*}$ is the indicator function of $(-\infty, 0] \times\{1\}$ and $g^{*}$ is the indicator function of $\{(1,0)\}$. Consequently, if $\rho \in$ $[0,1], u^{*} \in \mathbb{R}^{2}, v^{*} \in \mathbb{R}^{2}$ and $\rho u^{*}+(1-\rho) v^{*}=0$, then $\rho f^{*}\left(u^{*}\right)+(1-\rho) g^{*}\left(v^{*}\right)=\infty$, and so $(0.5)$ fails. We note that $(\mathcal{A B})$ is satisfied in this example because $g \in \mathcal{C C}\left(\mathbb{R}^{2}\right)$.

Remark 4. Let $f, g \in \mathcal{P C} \mathcal{L} \mathcal{S C}(E), f, g$ satisfy $(\mathcal{A B}), x \in E$ and $f(x)=g(x) \in \mathbb{R}$. We briefly discuss the problem of finding a formula for $\partial(f \vee g)(x)$. Suppose first that, for all $w^{*} \in \partial(f \vee g)(x)$, the following "exact" version of $(0.5)$ holds:

$$
(f \vee g)^{*}\left(w^{*}\right)=\min _{\rho \in[0,1], u^{*}, v^{*} \in E^{*}, \rho u^{*}+(1-\rho) v^{*}=w^{*}}\left[\rho f^{*}\left(u^{*}\right)+(1-\rho) g^{*}\left(v^{*}\right)\right] .
$$

Then it is easily seen that

$$
\partial(f \vee g)(x)=\operatorname{co}(\partial f(x) \cup \partial g(x))
$$

In general, we have the formulae for $(f \vee g)^{*}\left(w^{*}\right)$ given by (2.1) and (2.3), and we have the formula established by Volle in [8], Théorème 2, p. 848 that

$$
\partial(f \vee g)(x)=\operatorname{co}(\partial f(x) \cup \partial g(x))+N_{\operatorname{dom} f}(x)+N_{\operatorname{dom} g}(x),
$$


where " $N_{C}(x)$ " stands for the normal cone to $C$ at $x$. However, we do not know an easy way of deducing (4.2) from (2.1) or (2.3).

The BICONJUGATE OF A MAXIMUM

It is an easy consequence of the definitions that if $f \in \mathcal{P C} \mathcal{L S C}(E)$, then

$$
t^{* *} \in E^{* *}, f^{* *}\left(t^{* *}\right) \leq 0 \quad \text { and } \quad w^{*} \in E^{*} \quad \Longrightarrow \quad\left\langle w^{*}, t^{* *}\right\rangle \leq f^{*}\left(w^{*}\right) .
$$

Lemma 5. Suppose that $f, g \in \mathcal{P C} \mathcal{L S C}(E), f$ and $g$ satisfy $(\mathcal{A B})$ and also that $f^{* *}\left(t^{* *}\right) \vee g^{* *}\left(t^{* *}\right) \leq 0$.

(a) Let $w^{*} \in E^{*}$. Then $\left\langle w^{*}, t^{* *}\right\rangle \leq(f \vee g)^{*}\left(w^{*}\right)$.

(b) $(f \vee g)^{* *}\left(t^{* *}\right) \leq 0$.

Proof. (a) Let $\delta>0$. If $\left(\rho, \sigma, u^{*}, v^{*}\right) \in L\left(w^{*}, \delta\right)$, then, using (4.3),

$$
\begin{aligned}
\rho f^{*}\left(u^{*}\right)+\sigma g^{*}\left(v^{*}\right) & \geq \rho\left\langle u^{*}, t^{* *}\right\rangle+\sigma\left\langle v^{*}, t^{* *}\right\rangle \\
& =\left\langle\rho u^{*}+\sigma v^{*}, t^{* *}\right\rangle \\
& \geq\left\langle w^{*}, t^{* *}\right\rangle-\delta\left\|t^{* *}\right\| .
\end{aligned}
$$

Thus, taking the infimum over $\left(\rho, \sigma, u^{*}, v^{*}\right) \in L\left(w^{*}, \delta\right)$,

$$
\left(f^{*} \underset{\delta}{\wedge} g^{*}\right)\left(w^{*}\right) \geq\left\langle w^{*}, t^{* *}\right\rangle-\delta\left\|t^{* *}\right\|,
$$

and (a) now follows from Theorem 2 by letting $\delta \rightarrow 0$. (b) is immediate from (a).

Theorem 6. Suppose that $f, g \in \mathcal{P C} \mathcal{L S C}(E)$, and $f$ and $g$ satisfy $(\mathcal{A B})$. Then

$$
(f \vee g)^{* *}=f^{* *} \vee g^{* *} \text { on } E^{* *} \text {. }
$$

Proof. We first prove that if $t^{* *} \in E^{* *}$, then

$$
(f \vee g)^{* *}\left(t^{* *}\right) \leq f^{* *}\left(t^{* *}\right) \vee g^{* *}\left(t^{* *}\right) .
$$

Let $\alpha:=f^{* *}\left(t^{* *}\right) \vee g^{* *}\left(t^{* *}\right)$. Since (6.1) is immediate if $\alpha=\infty$, we can and will suppose that $\alpha \in \mathbb{R}$. Then (6.1) follows from Lemma 5(b) with $f$ replaced by $f-\alpha$ and $g$ replaced by $g-\alpha$.

Since $f \vee g \geq f$ on $E,(f \vee g)^{* *} \geq f^{* *}$ on $E^{* *}$. Similarly, $(f \vee g)^{* *} \geq g^{* *}$ on $E^{* *}$, and so $(f \vee g)^{* *} \geq f^{* *} \vee g^{* *}$ on $E^{* *}$. The result now follows from (6.1).

Corollary 7. Let $g_{0} \in \mathcal{P C} \mathcal{L S C}(E)$ and $g_{1}, \ldots, g_{m} \in \mathcal{C C}(E)$. Then

$$
\left(g_{0} \vee \cdots \vee g_{m}\right)^{* *}=g_{0}{ }^{* *} \vee \cdots \vee g_{m}{ }^{* *} .
$$

Proof. This is immediate from Theorem 6 and induction.

Remark 8. We now give an example showing that $(0.4)$ can fail when $(\mathcal{A B})$ is not satisfied, even if $f \vee g \in \mathcal{P C} \mathcal{L S C}(E)$. (The conclusion of Theorem 2 must also fail for this example, as we shall see in Theorem 12.) Let $E=c_{0}$,

$$
\begin{gathered}
C:=\left\{\left\{x_{n}\right\}_{n \geq 1} \in c_{0}: \quad x_{1} \geq x_{2} \geq x_{3} \geq \ldots 0\right\}, \\
D:=\left\{\left\{x_{n}\right\}_{n \geq 1} \in c_{0}: \sum_{n=1}^{\infty} \frac{1}{2^{n}}\left(x_{1}-x_{n+1}\right)=0\right\},
\end{gathered}
$$


and define $f, g \in \mathcal{P C} \mathcal{L S C}(E)$ by $f:=I_{C}$ and $g:=I_{D}$. Now if $x \in C \cap D$, then

$$
\sum_{n=1}^{\infty} \frac{1}{2^{n}}\left(x_{1}-x_{n+1}\right)=0 \text { and, for all } n \geq 1, \quad x_{1}-x_{n+1} \geq 0 .
$$

It follows that, for all $n \geq 1, x_{1}-x_{n+1}=0$, and so $x$ is a constant sequence. Since $x \in c_{0}$, we deduce that $x=0$. These observations lead easily to the conclusion that $f \vee g=I_{\{0\}}$, from which $(f \vee g)^{*}=0$ and $(f \vee g)^{* *}=I_{\{0\}}$ (relative to $\left.E^{* *}\right)$. In particular, if $e:=(1,1,1, \ldots) \in \ell^{\infty}=E^{* *}$, then

$$
(f \vee g)^{* *}(e)=\infty \text {. }
$$

If $m \geq 1$, define $y^{m}$ and $z^{m} \in E$ as follows:

$$
y_{n}^{m}:=\left\{\begin{array}{ll}
1 & \text { if } n \leq m ; \\
0 & \text { otherwise; }
\end{array} \quad \text { and } \quad z^{m}{ }_{n}:= \begin{cases}1 & \text { if } n \leq m ; \\
2 & \text { if } n=m+1 ; \\
0 & \text { otherwise. }\end{cases}\right.
$$

Then $y^{m} \in C$ and $z^{m} \in D$, from which $f\left(y^{m}\right)=0$ and $g\left(z^{m}\right)=0$. Using (0.1), we deduce from this that $f^{* *}\left(\widehat{y^{m}}\right)=0$ and $g^{* *}\left(\widehat{z^{m}}\right)=0$. Since $\widehat{y^{m}} \rightarrow e$ and $\widehat{z^{m}} \rightarrow e$ in the weak ${ }^{*}$ topology of $E^{* *}$ as $m \rightarrow \infty$, and $f^{* *}$ and $g^{* *}$ are weak ${ }^{*}$ lower semicontinuous, it follows that $f^{* *}(e) \leq 0$ and $g^{* *}(e) \leq 0$, from which

$$
\left(f^{* *} \vee g^{* *}\right)(e) \leq 0
$$

If we now combine (8.1) and (8.2), we see that (0.4) fails, as claimed.

\section{The PRECONJUGATE OF A MAXIMUM}

Lemma 9. Suppose that $f, g \in \mathcal{P C} \mathcal{L} \mathcal{S C}(F)$ with $\operatorname{dom} f^{*} \cap \operatorname{dom} g^{*} \neq \emptyset$, and $\delta>0$.

(a) Let $x^{*} \in F^{*}$. Then $f \wedge g \geq x^{*}-\left(f^{*} \vee g^{*}\right)\left(x^{*}\right)-\delta\left\|x^{*}\right\|$ on $F$.

(b) $f \wedge_{\delta} g: F \rightarrow \mathbb{R} \cup\{\infty\}$.

(c) $f \underset{\delta}{\wedge} g \leq f$ on $F$ and $f \underset{\delta}{\wedge} g \leq g$ on $F$.

(d) $f \underset{\delta}{\wedge} g$ is convex.

Proof. (a) Since the result is trivial if $\left(f^{*} \vee g^{*}\right)\left(x^{*}\right)=\infty$, we can and will suppose that $\left(f^{*} \vee g^{*}\right)\left(x^{*}\right) \in \mathbb{R}$. Let $w \in F$ and $(\rho, \sigma, u, v)$ be an arbitrary element of $L(w, \delta)$. Then

$$
\begin{aligned}
\rho f(u)+\sigma g(v) & \geq \rho\left[\left\langle u, x^{*}\right\rangle-f^{*}\left(x^{*}\right)\right]+\sigma\left[\left\langle v, x^{*}\right\rangle-g^{*}\left(x^{*}\right)\right] \\
& \geq\left\langle\rho u+\sigma v, x^{*}\right\rangle-f^{*}\left(x^{*}\right) \vee g^{*}\left(x^{*}\right) \\
& \geq\left\langle w, x^{*}\right\rangle-\delta\left\|x^{*}\right\|-\left(f^{*} \vee g^{*}\right)\left(x^{*}\right) .
\end{aligned}
$$

We now obtain (a) by taking the infimum over $(\rho, \sigma, u, v) \in L(w, \delta)$.

(b) This follows from (a) by taking $x^{*} \in \operatorname{dom} f^{*} \cap \operatorname{dom} g^{*}$.

(c) We shall prove that $f \underset{\delta}{\wedge} g \leq f$ on $F$, the proof that $f \underset{\delta}{\wedge} g \leq g$ on $F$ is similar. So let $w \in F$. We need to show that

$$
(f \underset{\delta}{\wedge} g)(w) \leq f(w)
$$

Since this is trivial if $f(w)=\infty$, we can and will suppose that $w \in \operatorname{dom} f$. Fix $v \in \operatorname{dom} g$. If $\rho>0, \sigma>0, \rho+\sigma=1$ and $(\rho, \sigma)$ is sufficiently close to $(1,0)$, then $(\rho, \sigma, w, v) \in L(w, \delta)$ and so $\rho f(w)+\sigma g(v) \geq\left(f \wedge_{\delta} g\right)(w)$. We now obtain (9.1) by letting $(\rho, \sigma) \rightarrow(1,0)$. 
(d) For $i=1,2$, let $w_{i} \in E, \lambda_{i}>0$ and $\sum_{i} \lambda_{i}=1$. Put $w_{3}:=\sum_{i} \lambda_{i} w_{i}$. We shall prove that

$$
\sum_{i} \lambda_{i}(f \underset{\delta}{\wedge} g)\left(w_{i}\right) \geq(f \underset{\delta}{\wedge} g)\left(w_{3}\right)
$$

which will give the required result. To this end, let $\left(\rho_{i}, \sigma_{i}, u_{i}, v_{i}\right)$ be arbitrary elements of $L\left(w_{i}, \delta\right)$. It is easy to check that

$$
\sum \lambda_{i}\left(\rho_{i} u_{i}+\sigma_{i} v_{i}\right) \in B\left(w_{3}, \delta\right) .
$$

Put $\rho_{3}:=\sum_{i} \lambda_{i} \rho_{i} \in(0,1), \sigma_{3}:=\sum_{i} \lambda_{i} \sigma_{i} \in(0,1), u_{3}:=\sum_{i} \lambda_{i} \rho_{i} u_{i} / \rho_{3} \in F$ and $v_{3}:=\sum_{i} \lambda_{i} \sigma_{i} v_{i} / \sigma_{3} \in F$. Since $\rho_{3}+\sigma_{3}=1$, it follows from these definitions that

$$
\sum_{i} \lambda_{i} \rho_{i} f\left(u_{i}\right) \geq \rho_{3} f\left(u_{3}\right) \quad \text { and } \quad \sum_{i} \lambda_{i} \sigma_{i} g\left(v_{i}\right) \geq \sigma_{3} g\left(v_{3}\right)
$$

Consequently,

$$
\sum_{i} \lambda_{i}\left[\rho_{i} f\left(u_{i}\right)+\sigma_{i} g\left(v_{i}\right)\right] \geq \rho_{3} f\left(u_{3}\right)+\sigma_{3} g\left(v_{3}\right) .
$$

We also derive from (9.3) that $\rho_{3} u_{3}+\sigma_{3} v_{3} \in B\left(w_{3}, \delta\right)$. Combining this with (9.4), we obtain

$$
\sum_{i} \lambda_{i}\left[\rho_{i} f\left(u_{i}\right)+\sigma_{i} g\left(v_{i}\right)\right] \geq(f \underset{\delta}{\wedge} g)\left(w_{3}\right),
$$

and (9.2) now follows by taking the infima over $\left(\rho_{i}, \sigma_{i}, u_{i}, v_{i}\right) \in L\left(w_{i}, \delta\right)$.

Lemma 10. Suppose that $f, g \in \mathcal{P C} \mathcal{L S C}(F)$ and $\operatorname{dom} f^{*} \cap \operatorname{dom} g^{*} \neq \emptyset$.

(a) Let $x^{*} \in F^{*}$. Then $x^{*}-f \hat{0}_{0} g \leq\left(f^{*} \vee g^{*}\right)\left(x^{*}\right)$ on $F$.

(b) $f \underset{0}{\wedge} g \leq f$ on $F$ and $f \underset{0}{\wedge} g \leq g$ on $F$.

Proof. These assertions follow easily from Lemma 9 by letting $\delta \rightarrow 0$.

Theorem 11. Suppose that $f, g \in \mathcal{P C} \mathcal{L} \mathcal{S C}(F)$ and $\operatorname{dom} f^{*} \cap \operatorname{dom} g^{*} \neq \emptyset$. Then

$$
f \hat{0} g \in \mathcal{P C} \mathcal{L S C}(F) \quad \text { and } \quad(f \underset{0}{\wedge} g)^{*}=f^{*} \vee g^{*} \text { on } F^{*}
$$

Proof. It is clear from Lemma 9 (a) by letting $\delta \rightarrow 0$ that $(f \underset{0}{\wedge} g): E \rightarrow \mathbb{R} \cup\{\infty\}$ and is convex. In order to show that $f \wedge g \in \mathcal{P C} \mathcal{L S C}(F)$, it only remains to prove that $f \hat{}_{0} g$ is lower semicontinuous on $F$. To this end, let $w \in F$ and $\alpha<(f \hat{0} g)(w)$. We can choose $\delta>0$ so that $\alpha<(f \underset{\delta}{\wedge} g)(w)$. Let $\eta:=\delta / 2$. Since

$$
x \in B(w, \eta) \quad \Longrightarrow \quad B(x, \eta) \subset B(w, \delta),
$$

it follows by taking the appropriate infima that

$$
x \in B(w, \eta) \quad \Longrightarrow \quad(f \underset{\eta}{\wedge} g)(x) \geq(f \underset{\delta}{\wedge} g)(w)
$$

Hence

$$
x \in B(w, \eta) \quad \Longrightarrow \quad(f \underset{0}{\wedge} g)(x)>\alpha .
$$

This gives the required lower semicontinuity. It follows from Lemma $10(\mathrm{~b})$ that $(f \underset{0}{\wedge} g)^{*} \geq f^{*}$ on $F^{*}$ and $(f \underset{0}{\wedge} g)^{*} \geq g^{*}$ on $F^{*}$, from which $(f \underset{0}{\wedge} g)^{*} \geq f^{*} \vee g^{*}$ on $F^{*}$. The opposite inequality follows by taking the supremum over $F$ in Lemma $10(\mathrm{a})$.

Theorem 12. Suppose that $f, g \in \mathcal{P C} \mathcal{L} \mathcal{S C}(E)$ and $\operatorname{dom} f \cap \operatorname{dom} g \neq \emptyset$. Then

$$
(f \vee g)^{* *}=f^{* *} \vee g^{* *} \text { on } E^{* *} \Longleftrightarrow(f \vee g)^{*}=f^{*} \hat{0}^{*} \text { on } E^{*}
$$


Proof. We first note that $\operatorname{dom} f^{* *} \cap \operatorname{dom} g^{* *} \neq \emptyset$; hence, from Theorem 11 with $F:=E^{*}$ and $f$ and $g$ replaced by $f^{*}$ and $g^{*}$,

$$
f_{0}^{*} \underset{0}{\wedge} g^{*} \in \mathcal{P C} \mathcal{L S C}\left(E^{*}\right) \text { and }\left(f_{0}^{*} \underset{\sim}{\wedge} g^{*}\right)^{*}=f^{* *} \vee g^{* *} \text { on } E^{* *}
$$

It is immediate from this that

$$
(f \vee g)^{*}=f^{*} \underset{0}{\wedge} g^{*} \text { on } E^{*} \quad \Longrightarrow \quad(f \vee g)^{* *}=f^{* *} \vee g^{* *} \text { on } E^{* *} .
$$

Now suppose that $(f \vee g)^{* *}=f^{* *} \vee g^{* *}$ on $E^{* *}$. From $(12.1),(f \vee g)^{* *}=\left(f^{*} \underset{0}{\wedge} g^{*}\right)^{*}$ on $E^{* *}$, and consequently

$$
(f \vee g)^{* * *}=\left(f_{0}^{*} \underset{\wedge}{\wedge} g^{* * *} \text { on } E^{* * *}\right.
$$

Since both $(f \vee g)^{*}$ and $\left(f_{0}^{*} \underset{\wedge}{\wedge} g^{*}\right)$ are in $\operatorname{PC} \mathcal{L S C}\left(E^{*}\right)$, it follows from (0.1) (with $E$ replaced by $\left.E^{*}\right)$ that

$$
(f \vee g)^{*}=f_{0}^{*} \underset{0}{\wedge} g^{*} \text { on } E^{*}
$$

as required.

\section{REFERENCES}

1. H. Attouch and H. Brézis, Duality for the sum of convex funtions in general Banach spaces, Aspects of Mathematics and its Applications, J. A. Barroso, ed, Elsevier Science Publishers, 1986, pp. 125-133. MR 87m:90095

2. K. Fan, Minimax theorems, Proc. Nat. Acad. Sci. U.S.A. 39 (1953), 42-47. MR 14:1109f

3. J.-P. Gossez, Opérateurs monotones non linéaires dans les espaces de Banach non réflexifs, J. Math. Anal. Appl. 34 (1971), 371-395. MR 47:2442

4. H. König, Über das Von Neumannsche Minimax-Theorem, Arch. Math. 19 (1968), 482-487. MR 39:1947

5. R. T. Rockafellar, On the maximal monotonicity of subdifferential mappings, Pac. J. Math. 33 (1970), 209-216. MR 41:7432

6. S. Simons, Critères de faible compacité en termes du théorème de minimax, Seminaire Choquet 1970/1971, no. 24, 5 pages. MR 57:17218

7. S. Traoré and M. Volle, On the level sum of two convex functions on Banach spaces, J. Convex Analysis 3 (1996), 141-151. MR 97m:46116

8. M. Volle, Sous-differential d'une enveloppe supérieure de fonctions convexes, C. R. Acad. Sci. Paris 317 (1993), 845-849. MR 94h:49035

Department of Mathematics and Statistics, University of Western Australia, NedLANDS 6907, AUSTRALIA

E-mail address: fitzpatr@maths.uwa.edu.au

Department of Mathematics, University of California, Santa Barbara, California 93106-3080

E-mail address: simons@math.ucsb.edu 